\title{
ESTUDIO COMPARATIVO DE LA EFICACIA DEL "HETRAZAN", SANTONINA Y HEXYLRESORCINOL EN EL TRATAMIENTO DE LA ASCARIDIASIS EN EL NINO
}

\author{
Dr. FNRIQUE: FANTA NIIFET.
}

Cittedras de Pediatría de los Yrofs. Artuto Bacza Goñi y Jutio Meneghello R.

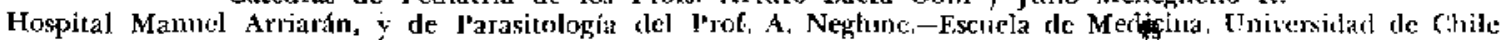

La ascaridiasis es una afección muy frecuente en los niños de nuestro país. Estudios epidemiológicos de Neghrne y cols. (12) demuestran que la prevalencia de esta parasitosis aumenta de norte a sur, adquiriendo en los caserios de las vecindades de los lagos y en la isla de Chiloé gran importancia por su elevada frecuencia, que llega hasta el $65 \%$ de los encuestados.

\section{Cuadro I}

Distribucion de la ascaridiasis en chile, por grupos de edad y por zonas, segin Neghtme y cols.

\begin{tabular}{|c|c|c|c|c|c|c|}
\hline \multirow[b]{2}{*}{ Tonas } & \multicolumn{3}{|c|}{ I a 6 años } & \multicolumn{3}{|c|}{7 a 14 años } \\
\hline & $\begin{array}{l}\text { Total } \\
\text { encuest. }\end{array}$ & $\begin{array}{c}\text { No } \\
+\end{array}$ & $\%$ & $\begin{array}{l}\text { Total } \\
\text { sncuest. }\end{array}$ & $\begin{array}{c}\text { No } \\
+\end{array}$ & $\%$ \\
\hline $\begin{array}{l}\text { Norte } \\
\text { Central } \\
\text { Sur }\end{array}$ & $\begin{array}{r}92 \\
284 \\
4.54\end{array}$ & $\begin{array}{r}2 \\
66 \\
309\end{array}$ & $\begin{array}{l}2,17 \\
23,23 \\
68\end{array}$ & $\begin{array}{r}210 \\
3.711 \\
629\end{array}$ & $\begin{array}{r}21 \\
1.015 \\
399\end{array}$ & $\begin{array}{l}10 \\
27,3 \\
63,45\end{array}$ \\
\hline
\end{tabular}

En encuestas personales realizadas entre habitantes ribereños de los Lagos Ranco y Rupanco, hemos encontrado hasta un $\mathbf{8 0 \%}$ de individuos parasitados con áscaris lumbricoides, sobre más de 1.000 individuos encuestados.
La infección de los niños se produce prineipalmente al jugar con tierra y llevarse las manos sucias a la boca; de ahí que en la prevención de esta parasitosis tengan enorme importancia las actividades de educación sanitaria, destinadas a la implantación de hábitos de prevención de la contaminación fecal del suelo y del agua, en la escuela $y$ en el hogar, y a la implantación de hábitos de higiene personal, tales como el lavado de manos después de trabajar o jugar con tierra $\mathrm{y}$ antes de comer.

La ascaridiasis, especialmente en el niño, debe tratarse siempre, porque, además de la sintornatologia que suele presentar, por demás vaga y proteiforme, puede producir complicaciones que requieren intervenciones de urgencia (obstrucción intestinal, obstrucción del colédoco; fenómenos asficticos por migración del gusano por anestésicos éter, cloroformo- o por tetracloroetileno).

La droga de elección, para niños mayores, es el hexylresorcinol. Sin embargo, tiene el inconveniente de su causticidad y de necesitar una dieta exenta de proteínas animales, lo que impide su empleo en todas las edades $y$ en campañas sanitarias. 
Medicamentos clásicos, como aceite de quenopodio, timol y santonina, son de relativa efectividad $y$ tienen un estrecho margen quimioterapéutico, por lo que suelen observarse manifestaciones graves de susceptibilidad personal con su uso. De ahí que se hayan ensayado diversas drogas en el tratamiento de la ascaridiasis del niño.

En 1949, Oliver González (13) introduce una nueva droga en el tratamiento de esta parasitosis, el "Hetrazán", clorhidrato de 1-dietilcarbamil-4 metil-piperazina, droga que ha sido ensayada ampliamente en el tratamiento de una helmintiasis, que no existe en nuestro país, la filariasis.

Antecediendo al empleo del Hetrazán en la ascaridiasis humana, Herwitt y col. (6-7) habian demostrado su eficacia sobre los áscaris del perro con $100 \%$ de curación.

Trabajos posteriores de Harned y col. (5) sobre la farmacología de este medicamento, precisaron su baja toxicidad y las escasas reacciones secundarias que producía.

En cuanto a la dosis, Oliver González usó 2 miligramos por kilogramo peso, en un día único de tratamiento; otros autores (2-3-8-911-14-15-19) utilizan entre 2 a 5 miligramos por kilogramo peso $y$ por dosis, dos a tres veces al día, durante 3 a 8 dias.

Ultimamente, varios investigadores han empleado el jarabe de "Hetrazán", preparado que contiene 30 miligramos de droga por cc., con resultados satisfactorios (11-8).

En la actualidad, la experiencia mundial en el tratamiento de la ascaridiasis con "Hetrazán" es numerosa, y todos los autores que lo han ensayado insisten en la baja toxicidad de la droga y su fácil administración, características que la hacen muy útil para ser empleada en lactantes y niños menores $y$ en actividades tendientes al control del áscaris lumbricoides.

Este trabajo tiene por objeto exponer 10 que hemos observado en el tratamiento de la ascaridiasis en el niño con santonina y hexylresorcinol, medicamentos conocidos entre nosotros, y con "Hetrazán", droga que aun no había sido ensayada en nuestro medio.

\section{Nuestra experiencia}

En forma preliminar, para conocer la tolerancia del "Hetrazán" ", efectuamos un estudio en enero de 1951, en 15 pacientes con ascaridiasis confirmada, en una campaña sanitaria, cuyo objetivo era la investigación de algunos helmintos.

Las edades de los pacientes fluctuaron entre 1 y 65 años. Usamos el "Hetrazán" en comprimidos de $\mathbf{5 0}$ miligramos, administrando por vía oral 3 a 4 miligramos por kilogramo de peso y por dosis, tres veces al día, antes de las comidas, en un día único de tratamiento. Los pacientes fueron controlados a las 24 horas después de realizada la terapéutica. La droga fué bien tolerada y no observamos signos graves de toxicidad inmediata.

\section{A. Material y método}

En el presente trabajo, el material está constituído por niños lactantes, preescolares y escolares, que consultaron a la Policlínica de Enfermedades Parasitarias de la Universidad de Chile por ascaridiasis, y por enfermos con la misma parasitosis internados en el Hospital Manuel Arriarán $\mathrm{y}$, anexo.

El diagnóstico en todos los casos fué certificado por el hallazgo de huevos de áscaris lumbricoides en exámenes de deposiciones efectuados por el método directo $y$ por el método de concentración de Teleman modificado.

I. Hetrazán. a) Siete preescolares de $2 \frac{1}{2}$ a 5 años se trataron con comprimidos de "Hetrazán" a razón de 20 mgrs. por kilogramo de peso diario, repartido en dos dosis antes de las comidas, durante 3 días. La forma de tratamiento fué ambulatoria, excepto un caso hospitalizado.

b) Quince nirios, entre 1 y 10 años de edad, recibieron la mismo dosis que el grupo anterior, pero durante 5 días. La terapéutica se hizo en forma ambulatoria.

c) En un tercer grupo de $\mathbf{5 2}$ niños, cuyas edades fluctuaron entre 1 año 4 meses y 11 años, empleamos "Hetrazán" * en jarabe, en un preparado que contiene 30 mgrs. por cc. El jarabe se administró en ayunas, por vía oral, durante 3 días, a razón de 20 mgrs. diarios de droga por kilogramo de peso. Treinta y seis niños fueron tratados ambulatoriamente y 16 niños recibieron su terapéutica estando hospitalizados.

En estos tres grupos el régimen alimentario fué el que habitualmente ingería el niกิo. 
Como criterio de eficacia del "Hetrazán" y de control de curación de la ascaridiasis, tuvimos la expulsión de lombrices -que siempre fueron llevadas a nuestro laboratorio- $y$ tres exámenes coprológicos efectuados por el método directo y por el de concentración (Teleman modificado) en un lapso de 9 días, una semana después de terminada la terapéutica. Adoptamos este criterio para evitar la posibilidad de error por Ia existencía de huevos residuales y porque las hembras de Ascaris lumbricoides dejan de eliminar huevos por varios días, por diversas causas.

II. Santonina. En 37 niños entre 1 año 7 meses y 12 años de edad, usamos la santonina en papelillos con agregado de lactosa, en cantidad de $1 \mathrm{cgr}$. por año de edad y por dosis, 3 papelillos en total, según el siguiente esquema:

1 papelillo en ayunas.

1 papelillo al acostarse.

1 papelillo en ayunas al día siguiente.

2 horas después de la última dosis, un purgante salino, generalmente 20 grs. de sulfato de magnesia.

El tratamiento se efectuó en forma ambulatoria, sin restricciones alimentarias $y$ como criterio de control de la térapéutica tuvimos la expulsión de Ascaris lumbricoides y exámenes coprológicos posteriores.

III. Hexylresorcinol. Usamos grageas queratinizadas de hexylresorcinol, en 25 niños cuyas edades fluctuaron entre 2 y 14 años de edad. El día anterior al tratamiento se indicó al paciente una comida liviana. La droga se administró en ayunas, por vía oral, en las siguientes dosis:
4 años
0,20 grs.
4- 6 años
0,40 grs.
6- 8 años
0,60 grs.
8-12 aก̃os
0,80 grs.

Durante el día de tratamiento el niño no debía ingerir proteínas animales.

La terapia se hizo en forma ambulatoria y se tuvo igual criterio de control de su eficacia que en el caso de la santonina.

\section{B. Resuttados}

Analizaremos por separado los resultados obtenidos con los tratamientos con "Hetrazán"; santonina y hexylresorcinol.

Los éxitos que apreciamos con las diversas terapéuticas los catalogamos con éxitos parciales y éxitos completos. Llamamos éxi- tos parciales aquellos en que hubo expulsión de parásitos con la terapéutica, pero en los que los exámenes coprológicos demostraron la persistencia de huevos de Ascaris lumbricoides en las heces o en los que no se efectuaron estos exámenes coprológicos posteriores al tratamiento. Consideramos éxitos completos, la eliminación de gusanos con controles de deposiciones después de la terapia, que revelan la ausencia de huevos de Ascaris Iumbricoides, o bien, solamente, la negatividad de los exámenes.

Analizados los resultados por grupos de edades no observamos diferencias apreciables imputables a la edad, por lo cual se presentan sin la discriminación de este factor.

I. Hetrazán. Considerada en forma global la terapéutica con "Hetrazán", de 74 casos sometidos al tratamiento, en $\mathbf{5 8}$ casos $-78,37 \%$ - se obtuvo alguna respuesta favorable. En 16 casos - 21,73\% - hubo fracaso absoluto de la terapia.

a) Con el esquema terapéutico de $\mathbf{2 0}$ mgrs. por kilogramo de peso diario durante 3 dias, de comprimidos de "Hetrazán". se obtuvo éxito parcial en el $42,85 \%$ de los casos y éxito completo en el $57,15 \%$. Entre los éxitos parciales se incluye un caso que expulsó lombrices y no tiene controles coprológicos posteriores.

b) Con el esquema terapéutico de iguales dosis diarias que el anterior, pero durante 5 días, se obtuvo un $46,66 \%$ de éxitos parciales y $33,33 \%$ de éxitos completos. Entre los éxitos parciales se incluyen 2 casos $\sin$ control coprológico posterior.

c) En el tercer grupo, tratado con jara. be de "Hetrazán", se obtuvo éxito parcial en el $53,84 \%$ de los casos y éxito completo en el $21,15 \%$. Se incluyen como éxitos parciales 10 casos en que no hubo control coprológico posterior.

\section{Cuadro II}

Resultados del tratamiento con "Hetrazan" en 7 " ninos con ascaridiasis

\begin{tabular}{|c|c|c|c|c|c|}
\hline \multirow{2}{*}{$\begin{array}{l}\text { Pautas } \\
\text { terapéticas }\end{array}$} & \multirow[b]{2}{*}{ Notrat. } & \multicolumn{2}{|c|}{$\begin{array}{l}\text { Exitos } \\
\text { parciales }\end{array}$} & \multicolumn{2}{|c|}{$\begin{array}{c}\text { Exitos } \\
\text { completos }\end{array}$} \\
\hline & & $\mathrm{No}^{\circ}$ & $\%$ & vo & $\%$ \\
\hline $\begin{array}{l}\text { Comp. } 20 \text { mgrs. } \\
\times 3 \text { dins } \\
\text { Comp. } 20 \text { mgrs. }\end{array}$ & 7 & 3 & 42.85 & 4 & 57,15 \\
\hline $\begin{array}{l}x \quad 5 \text { días } \\
\text { Jarabe } 20 \text { mgrs. }\end{array}$ & IS & 7 & 46,60 & 5 & 33.33 \\
\hline $\begin{array}{l}\text { x } 3 \text { días } \\
\text { Totales }\end{array}$ & $\begin{array}{l}52 \\
74\end{array}$ & $\begin{array}{l}28 \\
98\end{array}$ & $\begin{array}{l}53,84 \\
51,35\end{array}$ & $\begin{array}{l}11 \\
20\end{array}$ & $\begin{array}{l}21,55 \\
27,02\end{array}$ \\
\hline
\end{tabular}


El número de Ascaris lumbricoides expulsados fluctuó entre la unidad y 23 ejemplares. La mayoría fueron eliminados vivos, pero con la vitalidad disminuída, ya sea junto con los excrementos, ya sea espontáneamente.

Toxicidad. La tolerancia a la droga fué buena en la mayoría de los casos, excep. to en 3 pacientes en que hubo cuadros alarmantes durante el tratamiento.

En 43 pacientes se produjo alguna o varias de las siguientes molestias en el curso de la terapéutica: sed, cefalea, decaimiento, somnolencia, palidez, rechazo alimentario, náuseas, vómitos, dolores abdominales, pseudo-abdomen agudo; que no duraron más allá de 6 a 48 horas.

$$
\text { Cuaclio IIr }
$$

Relacion entro in pauta terapéutica y la tolerancia del "Hetrazán" en 74 nifos con ascaridiasis

\begin{tabular}{|c|c|c|c|}
\hline \multirow{2}{*}{$\begin{array}{l}\text { Pauta } \\
\text { terapéntica }\end{array}$} & \multirow[b]{2}{*}{ Notrat. } & \multicolumn{2}{|c|}{ Bucna tolerancia } \\
\hline & & No & $\%$ \\
\hline \multicolumn{4}{|l|}{ Comp. 20 mgrs. } \\
\hline $\begin{array}{l}x 3 \text { tlias } \\
\text { Comp. } 20 \text { mgrs. }\end{array}$ & 7 & 3 & 42,85 \\
\hline$\times 5$ días & 15 & 11 & 78,93 \\
\hline $\begin{array}{l}\text { Jarabc } 20 \text { mgrs. } \\
x 3 \text { dias ambulat. } \\
\text { Jarabe } 90 \text { mors. }\end{array}$ & 36 & 10 & 27,27 \\
\hline$\times \mathbf{3}$ días hosp. & 16 & 7 & 43,75 \\
\hline
\end{tabular}

Dos pacientes, de 7 y 9 años de edad, tratados con comprimidos durante 5 días, presentaron en el curso de la terapia cuadros de pseudo-abdomen agudo, que obligaron a su hospitalización de urgencia, e hicieron sospechar apendicitis y peritonitis; el cuadro cedió espectacularmente 12 horas después de iniciado, coincidiendo en ambos casos con la expulsión de gusanos (4).

En un lactante tratado con jarabe, por equivocación se dió una dosis 4 veces superior a la que le correspondía y se apreció el primer día palidez, gran decaimiento y somnolencia; al día siguiente se había recuperado.

II. Santonina. En $31-83,77 \%$ de los 37 pacientes sometidos a la terapéutica con santonina, hubo alguna respuesta favorable al tratamiento. En 6 casos $-22,33 \%-$ hubo fracaso absoluto de la terapia.

Se obtuvo éxito parcial en el $59.45 \%$ de los casos y éxito completo en el $24,32 \%$. Se incluyen entre los éxitos parciales, $10 \mathrm{ca}$ sos que expulsaron áscaris $\mathrm{y}$ no tienen controles coprológicos posteriores.

Toxicidad. La droga fué bien tolerada generalmente; 8 pacientes $-21,63 \%$ - tuvieron alguna o varias de las siguientes molestias en el curso de la terapéutica: cefalea, dolor abdominal, diarrea.

III. Hexylresorcinol, En $21-84 \%-$ de los 25 pacientes sometidos a la terapia con hexylresorcinol se obtuvo alguna respues. ta favorable. En 4 casos $-16 \%$ - hubo fra. caso completo al tratarniento.

Se obtuvo éxito parcial en el $32 \%$ y éxito completo en el $52 \%$. Se incluyen entre los éxitos parciales 4 casos que expulsaron gusanos y no tienen controles coprológicos posteriores.

Toxicidad. En general, la tolerancia a la droga fué satisfactoria y 5 pacientes $20 \%$ - tuvieron alguna o varias de las siguientes molestias en el curso del tratamiento: cefalea, somnolencia, dolor abdominal, diarrea.

\section{Comentario}

Para precisar la eficacia de las diversas crogas que empleamos en nuestro estudio, efectuamos un análisis comparativo:

\section{Cuadm JV}

Cuadro comparatirn de los resultados con tratamiento con "Hetrazán", Hexylresorrinol y Sanfonine on "136 nir̄ot ron nstaridinsis

\begin{tabular}{|c|c|c|c|c|c|c|c|}
\hline \multirow[b]{2}{*}{ Droga } & \multirow[b]{2}{*}{ No } & \multicolumn{2}{|c|}{$\begin{array}{c}\text { Exitos } \\
\text { parciales }\end{array}$} & \multicolumn{2}{|c|}{$\begin{array}{c}\text { Fxitos } \\
\text { completos }\end{array}$} & \multicolumn{2}{|c|}{ Fracasos } \\
\hline & & No & $\%$ & No & $\%$ & $N$ & $\%$ \\
\hline \multirow{3}{*}{$\begin{array}{l}\text { Hetracáu } \\
\text { Samtonina } \\
\text { Hexylresor- } \\
\text { rinol }\end{array}$} & 34 & $3 N$ & 51,35 & 20 & 27,02 & 16 & 21,62 \\
\hline & 32 & 22 & 59.45 & 9 & 24,32 & 6 & 16,48 \\
\hline & 25 & 8 & 32 & 13 & 52 & 4 & 16 \\
\hline
\end{tabular}

Realizado el estudio estadístico mediante el método de chi cuadrado, considerando los porcentajes de fracasos en relación con los éxitos parciales más los éxitos completos, no se aprecian diferencias significativas.

En el cuadro IV aparece evidente la acción antihelmíntica más eficaz del hexylresorcinol, quedando en un segundo plano, en forma más o menos similar, el hetrazán y la santonina.

Debemos llamar la atención de las dife- 
rencias notables entre nuestras cifras y las de autores extranjeros, que dan resultados muy superiores. Así, Hoekenga (8) y Lamson y col. (10) obtienen con el "Hetrazán" y hexylresorcinol $80 \%$ de curación con cada droga, respectivamente. Claro está, que cierto número de nuestros casos dados solamente como éxitos parciales, pueden haber curado de su ascaridiasis, con lo que nos acercaríamos algo a las tan distintas cifras de los autores extranjeros.

Aunque no tenemos bases pard explicar estas diferencias, se podría sugurir la pasibilidad de que las cepas dé Ascaris lumbricoides en Chile sean más resistentes a la terapéutica. Por otra parte, no sabemos qué valor tenga la presencia de pocos o múltiples ejemplares de este parásito, ya que la mayoría de los autores extranjeros trabajan con infestaciones intensas.

No debemos olvidar tampoco que nuestro método de control de la terapéutica es el examen seriado de deposiciones, el cual da un margen bastante amplio de seguridad de que el enfermo ha sanado, afirmación que no se puede realizar cuando se usa el método de conteo de huevos. Con ambos métodos escapan al control aquellos casos en que persisten después del tratamiento ejemplares machos.

El bajo número de éxitos parciales del hexylresorcinol con respecto a las otras dos drogas, podría tener su explicación en el hecho de que a menudo los áscaris son eliminados destruídos $\mathrm{y}$ no se les puede reconocer, lo que se debería a la acción destructora de este medicamento sobre la cutícula del parásito.

Nos queda por analizar el punto relativo a la tolerancia de los diversos medicamentos utilizados, para lo cual también efectuamos un estudio comparativo.

Vemos en este cuadro que el hexylresorcinol y la santonina han sido muy bien to-

Cuadro $V$

Cisadro comparation de la toleroncia al "Hetrazán", Hexvlresorcinol y Santomina en 736 niñss con ascaridianis

\begin{tabular}{|c|c|c|c|}
\hline \multirow[b]{2}{*}{ Dioga } & \multirow[b]{2}{*}{ Notrat. } & \multicolumn{2}{|c|}{$\begin{array}{c}\text { Buena } \\
\text { tolerancia }\end{array}$} \\
\hline & & No & $\%$ \\
\hline Hetrazán (jarabe) & 52 & 17 & 32,69 \\
\hline Hetrazán (comprimidos) & 22 & i4 & 69,63 \\
\hline Santonina & 37 & 29 & 78,87 \\
\hline Hexylresorcinol & $2 \sqrt{3}$ & 90 & 80 \\
\hline
\end{tabular}

lerados en la gran mayoría de los enfermos. Sin embargo, es necesario destacar que el hexylresorcinol tiene el inconveniente de que al ser masticado puede producir quemaduras superficiales de las mucosas, lo que prácticamente lo hace inaplicable en niños menores.

La santonina, por su parte, está descrita en todos los textos como un medicamento tóxico y se han publicado diversas comunicaciones de casos graves producidos por la terapia con este medicamento (1); $\sin \mathrm{em}-$ bargo, en la experiencia de 15 años de la Cátedra de Parasitología de la Universidad de Chile, sólo se han observado dos casos alarmantes de intoxicación con santonina.

Hemos dejado para el final el comentario sobre el "Hetrazán", pues nos merece ciertas consideraciones especiales.

El "Hetrazán", a las dosis de 2 a 3 miligramos por kilogramo de peso, es totalmente inocuo; y según Romaña (14), utilizando la dosis de 13 miligramos por kilogramo de peso durante 7 días, se obtienen buenos resultados. Basados en este antecedente pensamos en la posibilidad de disminuir los días de tratamiento, aumentando más la dosis, de donde nació el esquema adoptado por nosotros en este trabajo.

Con las altas dosis empleadas, hemos observado signos tóxicos; fuera de los 2 casos de pseudo-abdomen agudo ya descritos, recordemos que puede producir sed, cefalea, decaimiento, somnolencia, palidez, rechazo alimentario, náuseas, vómitos y dolores abdominales: molestias que en su mayor parte han sido descritas por otros autores, usando dosis elevadas.

Aunaue nuestra experiencia es limitada, del análisis de nuestra casuística podemas concluir:

1. El "Hetrazán" no demostró una eficacia terapéutica superior a la de otros medicamentos clásicos.

2. Manifestaciones de toxicidad fueron más frecuentes que las observadas con el empleo de santonina y hexylresorcinol.

A pesar de estos hechos, estimamos que el "Hetrazán" puede ser un elemento valioso en el arsenal terapéutico en contra de la ascaridiasis, especialmente en aquellos casos en los cuales no es posible utilizar los otros medicamentos. Creemos, además, que el alto porcentaje de manifestaciones de intolerancia se debe a la alta dosis de medicamento utilizada en nuestros casos. 


\section{Resumen y conclusiones}

1. Se tratan 136 niños con ascaridiasis con "Hetrazán", Santonina y Hexylresorcinol.

2. Con las dosis empleadas se obtiene con el "Hetrazán" un $51,35 \%$ de éxitos parciales y 27,02 de éxitos completos; con la Santonina se obtiene un $\mathbf{5 9 , 4 5 \%}$ de éxitos parciales y $24,32 \%$ de éxitos totales; y con el Hexylresorcinol se logra un $32 \%$ de exitos parciales y un $52 \%$ de éxitos totales.

3. El "Hetrazán" podría ser un medicamento de utilidad en aquellos casos en que los medicamentos clásicos no pueden ser utilizados.

4. Se observaron entre un $46,7 \%$ y $77,21 \%$ de manifestaciones tóxicas con e1 "Hetrazán". Entre las manifestaciones tóxicas observadas, cabe destacar cuadros pseudo-abdomen agudo, que pueden inducir a errores diagnósticos.

5. Las manifestaciones tóxicas con "Hetrazán" en nuestra serie, son seguramente atribuibles a las altas dosis empleadas.

\section{Summary and conclusions}

136 children with ascaridiasis are treated with "Hetrazan", Santonina and Hexylresorcinol.

With the doses used, a $51,35 \%$ of partial successes, and a $27,02 \%$ of complete stuccesses is obtained with Hetrazan, with Santonina a $59,45 \%$ and a $24,32^{r} \%$, and with Hexylresorcinol a $32 \%$ and a $52 \%$.

Hetrazan could be a useful drug in those cases in which the classical medication cannot be used.

A $46,7 \%$ and $77,21 \%$ of toxic manifesta- tions with Hetrazan were observed. Amongst the toxic manifestations seen, they mention 4 cases of pseudo acute abdomen that had diagnostical errors.

The toxic manifestations in our series can probably be attributed to the high dosage given.

\section{BIBLIOGRAFIA}

1. DESOILLE, H.-Rev, d'Oto-Neuro-Optht. 15: 170 173, 1937.

2. EDITORIAL,-Rev. San. c Hig. Pub. 25: 497.498, I95I.

3. ETEEDORI, J. y CRAWFORD, L..J. A. M. A. 143: 79 7.799, 1950 .

4. FANTA, E.-Bol. Inf. Par. Chilenas 7: 64-65. 1952.

5. HARNED, B., CUNNJNGHAM, R, HALLIDAX, S., VESSEY, R., YUDA, N., CLARK, M., HINE. C., COSGROVE, R. Y SUBBA ROW, J.-J. Lab. y Clin. Méct. 33: 216-295, 1948.

6. HEWITT, R., KLISHNER, S., STEWART, H., WHITE, E., WALLACE, W. Y SUBBA ROW, Y.J. Lab, y Clin. Med. 32: 1314-1329. 1947.

7. HEWITT, R, WALLACE, W, WHITE, E. Y SUBBA ROW, Y,-Jour of Pen. 34: 237-239. 1948.

8. HOECKENGA, M-Am. Jour, of Med. and Hig. I: $688-692,1952$

9. JASWANT SINC, N.. RAGHARAN, N., MISRA, B., KRISHNASWAMY, A. y RADHAGVINDA ROY.-Indian Med. Ga7. 87: 353-356, 1952.

10. SAMSON, P., BROWN, H., ROBBINS, B. y WARD, G-Am. Jour. of Hig. 15: 803-822, 1931.

11. LOLGHLIN, E., JOSEPH, A., RAPPAPORT, I. y MILLIN, W.-Lancer 261: 1197-1200.

12. NeghMF. A. y SILVA, R.-Rcy. Méd, de Chile 79: 449-457, 1951 .

18. OLIVER GONZALEZ, J.4 SANTJAGO STEVENSON, D. Y FIEWITT. R-South Alcd, Jour. 42; 6.5-66, 1949

14. ROMANAA, C., TORANZO, L. y MARCOLONGO, R,-An. In. Med. Rog. Liniv, Tucumán 3: 153I56. 1951 .

15. RUiz, REYES, t-Lol. lipid. (México) 13: 93, 1949.

RUI7. REYES, 7"., 'TORRES MUNOZ, 4. y CFRVANTîs GARCIA, L-Rev. Pal. y Médl. Tmp. (México) 2: $35-39,1950$. 